Mots. Les langages du politique

$72 \mid 2003$

La ville, entre dire et faire

\title{
L'aménagement urbain britannique entre participation locale et partenariats
}

Town planning in britain between community participation and partnership La urbanización británica entre participatión local y asociaciónes

Keith Jacobs

\section{OpenEdition}

Journals

Édition électronique

URL : https://journals.openedition.org/mots/6233

DOI : $10.4000 /$ mots. 6233

ISSN : 1960-6001

Éditeur

ENS Éditions

Édition imprimée

Date de publication : 1 juillet 2003

Pagination : 61-74

ISBN : 2-84788-034-8

ISSN : 0243-6450

\section{Référence électronique}

Keith Jacobs, «L'aménagement urbain britannique entre participation locale et partenariats », Mots. Les

langages du politique [En ligne], 72 | 2003, mis en ligne le 29 avril 2008, consulté le 23 avril 2022. URL http://journals.openedition.org/mots/6233 ; DOI : https://doi.org/10.4000/mots.6233

\section{(C) ENS Éditions}




\section{L'aménagement urbain britannique entre participation locale et partenariats}

Les réaménagements ${ }^{\circ \circ}$ des anciennes houillères situées non loin de Douvres et des chantiers navals de Chatham englobent de nombreux aspects des initiatives d'aménagement urbain en cours au Royaume-Uni. Ces deux projets peuvent servir à l'exploration d'un certain nombre de questions de politique d'urbanisme qui y sont liées. Par exemple, quel est l'impact sur le partenariat local des discours officiels, tels qu'ils sont médiatisés par les agences gouvernementales? Quelles tensions naissent au sein de partenariats qui visent à réconcilier les objectifs des acteurs locaux, des entreprises et du gouvernement?

Lorsque l'on s'engage dans une recherche portant sur une politique donnée, il est important de ne pas céder à la tentation d'interpréter littéralement les textes et les perceptions des acteurs. On devrait au contraire se focaliser sur l'exploration des significations latentes, sur l'évaluation des différentes lignes politiques et la recherche d'explications alternatives. C'est pour ces raisons que j'ai adopté une méthodologie de recherche influencée par une analyse critique de discours s'appuyant indirectement sur les travaux de Fairclough ${ }^{1}$. L'analyse de discours, en se concentrant sur la langue, offre une méthodologie d'analyse du processus politique

\footnotetext{
○ Politiste, Université de Tasmanie, Australie, Keith.Jacobs@utas.edu.au.

${ }^{\circ}$ Liste des termes étudiés dans cet article: participation locale (community participation), partenariats (partnerships), équilibre (balance), renforcement des capacités (capacity-building), entreprise locale (community enterprise), régénération économique (economic regeneration), infrastructure (infrastructure), investissement étranger (inward investment), marketing (marketing), mission (mission), zone mixte (mixed-use zone), revalorisation de l'espace (physical renewal), qualité de vie (quality of life), exclusion sociale (social exclusion), régénération durable (sustainable regeneration), directif (top down).

1. N. Fairclough, 1989, Language and power, Longman; 1992, Discourse and social change, Polity press; 1995, Critical discourse analysis, Longman.
} 
radicalement différente des autres. L'argument clé des théoriciens du discours est que la langue ne se réduit pas à la représentation d'une réalité sous-jacente, mais qu'elle est un prisme à travers lequel nous conceptualisons le monde. En d'autres termes, la langue elle-même est essentielle dans la manière dont nous donnons sens à la réalité. La tâche de l'analyse de discours est de ce fait généralement comprise comme la classification des manières dont les acteurs clés emploient une terminologie spécifique, écrite ou orale, au sein d'un contexte social et politique plus large.

On examinera tout particulièrement dans cet article la manière dont sont conçus et formulés les documents et les discours des politiques urbaines $^{2}$. Mon argumentation est la suivante: les stratégies adoptées par les acteurs dans deux localités visent à promouvoir de façons différentes une version de la réalité qui rassure des publics spécifiques. Les documents destinés à la promotion de la zone des houillères dans le Kent cherchent ainsi à fournir la preuve que l'investissement social est une réussite tout en démontrant dans le même temps que les politiques sont appliquées dans le cadre de partenariats avec les acteurs de la communauté alentour. Dans le cas de Chatham, les documents sont publiés afin de persuader les investisseurs de choisir le projet d'aménagement de Chatham Maritime pour y faire un nouvel investissement.

\section{Les gisements de houille de l'East Kent}

Le Kent ${ }^{3}$ est l'un des comtés les plus prospères du Royaume-Uni. La proximité de Londres et du continent européen, l'accès à des liaisons routières, et une campagne attrayante ont renforcé la compétitivité de cette région. Cependant, malgré une prospérité accrue, il reste des localités qui n'ont pas encore récolté les fruits de l'amélioration générale. Cela tient évidemment à la nature des changements économiques qui génèrent des inégalités entre et dans les localités ${ }^{4}$. L'impact du déclin industriel est particulièrement apparent dans les anciens villages miniers du Kent situés

2. Les documents rassemblés dans le cadre de ce travail de recherche comprennent des publications du département ministériel chargé de la politique régionale en GrandeBretagne, la SEEDA (South East of England development agency), et de Medway council.

3. Le comté du Kent couvre une zone de $3739 \mathrm{~km}^{2}$, avec une population de 1551000 habitants dont $60 \%$ résident en zone urbaine.

4. R. Hudson et A.Williams, 1995, Divided Britain, $2^{\mathrm{e}}$ edition, Wiley. 
non loin de Douvres. La production de houille a pris fin dans cette zone en 1989 et celle-ci n'a alors reçu qu'une aide limitée de la part du gouvernement. C'est seulement après une campagne de lobbying organisée par le Coalfield communities campaign (grand groupe de pression du Royaume-Uni) que l'aide régionale a été engagée en utilisant par exemple l'initiative RECHAR de la Commission européenne ${ }^{5}$ en 1989 et le Groupe d'action des houillères (Coalfield task force) en 1998.

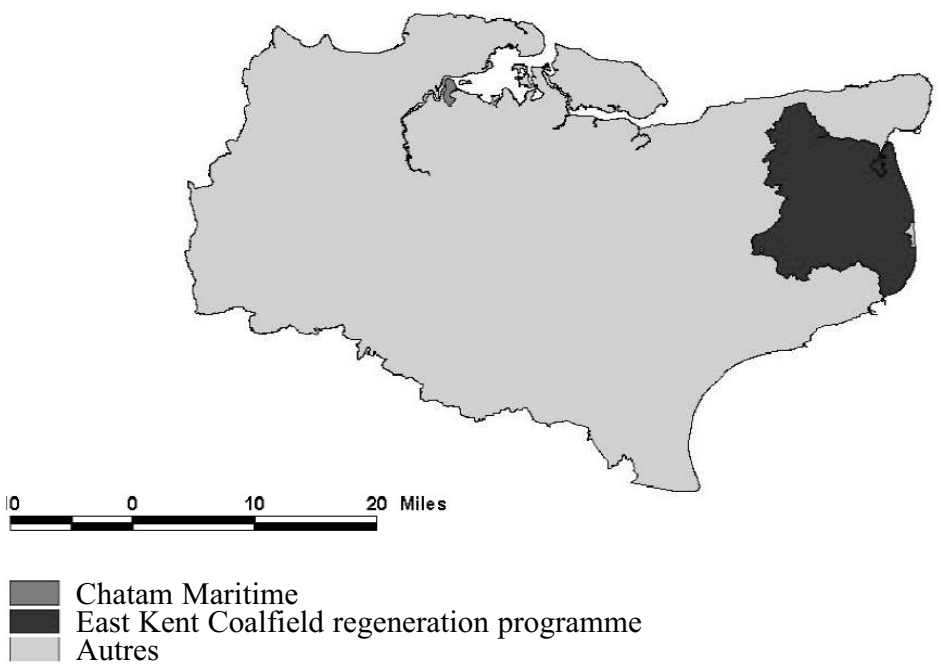

Figure 1: Comté du Kent. On voit la localisation de l'ancienne zone d'extraction de la houille et du réaménagement de Chatham Maritime

À leur apogée, les houillères employaient 11000 personnes; le dernier puits en activité, jugé insuffisamment rentable, a été fermé à la fin des années 1980. L'arrêt des houillères a eu une incidence majeure autant sur les résidents que sur les infrastructures. Le niveau de pauvreté et le taux

5. L'initiative communautaire RECHAR a été adoptée par la Commission européenne en 1989 pour soutenir la reconversion économique des anciennes zones d'extraction de la houille. 
de chômage sont très élevés dans les anciens villages miniers. L'organisme chargé de la régénération locale et de la revalorisation de l'espace est le EKCP, Partenariat pour la régénération des houillères du East Kent (East Kent coalfield regeneration partnership). Ce programme de 15 millions de livres est coordonné par le conseil municipal en partenariat avec des représentants de la communauté des résidents et du secteur associatif. Il est en place depuis 1997 et prendra fin en 2006.

\section{La participation locale (community participation) ${ }^{6}$}

L'EKCP se réunit toutes les six à huit semaines. Participent à ces réunions des représentants d'organismes tels que le Kent county council (Conseil du comté du Kent), l'autorité de police, et l'Aylesham community trust. L'un des aspects les plus remarquables du projet est l'ampleur avec laquelle la participation locale a été mise en valeur par les acteurs, ce à quoi s'ajoute la perception largement répandue parmi eux que l'EKCP a pleinement réussi à intégrer cette notion.

Cette mise en avant des aspects sociaux et communautaires constitue l'une des principales caractéristiques des accords de partenariat actuels. En effet, les projets de partenariat entrainent généralement un effort des décisionnaires pour s'engager auprès des habitants. Il y a un certain nombre de raisons à cela. C'est tout d'abord une manière d'assurer une légitimité au projet et de bâtir une logique formelle d'intervention étant donné que les habitants actifs et les acteurs politiques en font découler des modes d'organisation concrets. Ensuite, c'est à travers la participation locale que l'on peut juger du succès d'un projet selon les critères du programme de Budget unique de régénération SRB (Single regeration budget). Il n'est donc pas surprenant que les acteurs travaillant sur le terrain l'encouragent activement. La participation locale est le corollaire de la rénovation physique (physical renewal) et l'on peut affirmer que l'importance donnée à cette approche au sein de l'EKCP reflète les difficultés rencontrées pour garantir les investissements du secteur privé et la revalorisation de l'espace aux premières phases du projet.

La section suivante présente une analyse critique de la proposition de Budget unique destiné à la régénération $(\mathrm{SRB})$. Cela implique une lecture très précise afin de pouvoir mettre en évidence la manière dont le texte

6. Les acteurs politiques du Royaume-Uni emploient fréquemment ce terme pour désigner leur volonté de s'engager dans des pratiques de délégation du pouvoir décisionnel. 
remplit une fonction stratégique essentielle dans les pratiques de rénovation urbaine. Le texte établit un paradigme général qui sert à l'ensemble des activités figurant dans le projet, garantissant ainsi que les organisations et les acteurs se conforment aux modes opératoires attendus.

\section{Single regeneration budget: troisième appel d'offres, 1996-2003}

Comme il a été dit, l'offre du Dover district council a visiblement un objectif stratégique fondamental: obtenir des financements au projet. En tant que telle, cette offre s'adresse avant tout aux décisionnaires clés qui ont la responsabilité de déterminer à quelle proposition seront attribuées les ressources. Son but est de remplir les critères requis pour obtenir des fonds. Ce qui signifie que la proposition doit être conforme aux exigences exposées dans le Single regeneration bidding guidance (littéralement: Guide destiné aux offres de régénération unique $)^{7}$. Les auteurs de la proposition doivent de fait démontrer que les projets qui nécessitent un financement correspondent aux objectifs généraux du gouvernement. Il n'est donc pas surprenant que le document soit soigneusement formulé afin de susciter une réaction favorable de la part des décisionnaires.

La proposition East Kent coalfields SRB3 submission, comme d'autres rapports de ce type, en est un bon exemple. L'aspect compétitif des programmes d'offre du SRB n'est-il pas un instrument politique qui permet de garantir que les objectifs du gouvernement seront globalement pris en compte? La question se pose. On demande en effet aux responsables du gouvernement de noter chaque offre selon des critères exposés dans les directives ${ }^{8}$. En pratique, cela signifie que les critères sont axés sur des problèmes liés à la gestion de chaque partenariat plutôt qu'à un contenu politique. Mawson et Hall expliquent qu'en pratique chaque offre cherche à anticiper ce qui est perçu comme les priorités de chaque Government office for the region (direction régionale) ${ }^{9}$.

L'introduction de ce rapport donne les grandes lignes de la mission (mission) générale du programme de régénération.

7. Department of environment, transport and the regions, 1999, SRB bidding guidance round 5 South East single regeneration budget, London, DETR.

8. J. Mawson et S. Hall, 2000, Joining it up locally? Area regeneration and holistic government in England in regional studies, vol. 34, $\mathrm{n}^{\circ} 1$ p. 67.

9. Mawson et Hall affirment que la majeure partie de ces informations a été glanée auprès d'un fonctionnaire qui travaillait au sein de la Direction régionale. 
Le Programme de régénération des houillères de l'East Kent s'engage à renforcer la capacité des organisations et des groupes locaux (community) à mettre en place leurs structures, systèmes, personnels et qualifications, leur permettant ainsi de mieux définir et de mieux atteindre leurs objectifs, de s'engager dans la consultation et la planification, de gérer les projets locaux et de participer (participate) à des partenariats (partnerships) et à des entreprises locales (community enterprises). Ce programme vise également à poursuivre l'élaboration d'une bonne pratique, à ajouter de la valeur et à soutenir le développement des partenariats existants tout en ayant pour but l'amélioration de la qualité de vie (quality of life) de la communauté locale (East Kent coalfields regeneration programme, 1999, Single regeneration budget challenge fund-Round 5. Dover district council, p. 3).

La présentation de la mission résume en un seul paragraphe toute la stratégie du programme. Il n'y a rien qui puisse choquer dans cet exposé, d'ailleurs représentatif de nombreuses autres présentations de mission préparées pour le SRB. La mission est vraiment une mission de renforcement des capacités (capacity building) qui apporte un soutien aux groupes et aux individus dans le but d'améliorer «la qualité de vie de la communauté locale». C'est un exposé de «ce que l'on peut faire» qui laisse entendre qu'une action collective peut faire la différence. L'exposé est délibérément écrit dans un langage consensuel afin de suggérer que ce qui est proposé n'est pas sujet à controverse et mérite de fait d'être soutenu indépendamment des affiliations politiques ou des idéologies. Cependant, à y regarder de plus près, les termes employés dans l'exposé sont très souvent des concepts contestés. Par exemple, l'emploi de partenariat et de communauté locale (community) implique un consensus et un accord. Or, il y a régulièrement des conflits marqués, non seulement entre les différents partenaires, mais également au sein même des communautés locales (communities). L'exposé de mission présente l'intervention politique comme quelque chose d'implicite et de non problématique.

La proposition de régénération bénéficie du soutien d'une coalition très vaste qui comprend les dirigeants des conseils des autorités locales, le secteur associatif, les représentants des entreprises, des forums de jeunes et des établissements éducatifs. Encore une fois, il est censé signifier qu'un partenariat substantiel est en place, liant le secteur public et le secteur privé et méritant de ce fait l'attribution de ressources gouvernementales.

Si on se reporte aux buts et objectifs stratégiques, ils sont, tout comme l'exposé de mission, censés rassurer sur le plan symbolique. Ces objectifs 
ne sont nullement polémiques. Ils paraissent tous conformes aux propres buts stratégiques du gouvernement ${ }^{10}$. Ils visent à :

- accroitre les perspectives d'emploi, l'éducation et les qualifications des habitants;

- traiter le problème de l'exclusion sociale (social exclusion) et accroitre les opportunités d'emploi pour les plus défavorisés;

- promouvoir la régénération durable (sustainable regeneration), l'amélioration et la protection de l'environnement et des infrastructures (infrastructures) ;

- soutenir et promouvoir la croissance dans les communautés (communities) et les entreprises locales;

— traiter le problème de la criminalité et de la drogue, et améliorer la sécurité de la communauté.

(East Kent coalfields regeneration programme, 1999, Single regeneration budget challenge fund - Round 5. Dover district council, p. 3).

Les directives de l'appel d'offres 5, publiées par le ministère de l'Environnement, des transports et des régions, le DETR (Department of environment, transport and the regions), exposent ce que l'on attend d'une offre. Par exemple, le paragraphe 12 stipule:

L'expérience montre que les projets les plus innovants ont une inscription géographique claire, s'appuient sur une recherche détaillée et un engagement actif auprès des résidents pour la détermination d'une zone d'action et sont proposés par un partenariat à base large. L'engagement effectif de partenariats concrets en faveur du renforcement des capacités locales constituera un critère de soutien ${ }^{11}$.

Il n'est pas surprenant que les offres retenues soient très orientées vers la communauté locale et incluent dans le programme des projets de participation des habitants. Cet exemple illustre en quoi de tels textes remplissent une fonction stratégique vitale. La coalition des intérêts réunis pour promouvoir l'initiative de régénération des houillères du

10. En fait, ils sont exactement semblables à ceux du gouvernement. Cet effet de miroir par lequel les organisations intègrent l'ensemble des objectifs du gouvernement est symptomatique du pouvoir que détient le gouvernement central par rapport aux initiatives de régénération locale.

11. Department of environment, transport and the regions, 1999, SRB bidding guidance round 5 South East single regeneration budget, London, DETR. 
East Kent est typique de nombreuses autres offres qui cherchent à obtenir des fonds gouvernementaux. Les textes décrivant les politiques urbaines sont importants car ils renforcent l'impression qu'il y a un consensus culturel au sein de toute la communauté concernée. À l'intérieur de ce paradigme hégémonique, les conflits idéologiques sont rarement articulés puisqu'il n'y a en fait aucun espace discursif dans lequel ils peuvent se déployer. Au contraire, le lancement d'une véritable politique est limité par ce que s'imposent à eux-mêmes les acteurs clés: ils sont tous conscients que la reconnaissance d'un désaccord ou d'une tension réduira leurs chances d'obtenir des fonds. C'est pourquoi les conflits qui se manifestent publiquement tendent à tourner autour de problèmes de personnes et de modes de gestion plutôt que d'objectifs politiques.

\section{Le projet d'aménagement de Chatham Maritime}

Chatham Maritime se situe dans les limites du territoire de Medway council qui inclut également les villes de Rochester et de Gillingham. La population de Medway est de 240000 habitants. Chatham Maritime est le plus grand projet de réaménagement du Sud-Est de l'Angleterre et a bénéficié d'un investissement de 144 millions de livres via English partnerships ${ }^{12}$ et de six millions de livres de la part de son successeur, la South East of England development agency (SEEDA) ${ }^{13}$. Il s'y ajoute l'investissement de 300 millions de livres par le secteur privé, ce qui a donné lieu à la construction d'une marina d'une capacité de mouillage de 175 voiliers, de 250 maisons et de nouveaux espaces de bureaux d'environ 2800 mètres carrés. Le terrain occupe 146 hectares du site des Royal Dockyards qui a fermé en 1984. La fermeture des docks a entrainé la perte de 7000 emplois et d'un nombre équivalent dans les entreprises associées aux docks ${ }^{14}$.

12. English partnerships est l'agence gouvernementale responsable du financement des projets d'infrastructures. Un grand nombre de ses responsabilités ont été transférées aux Agences de développement régional (Regional development agencies).

13. L'Agence d'aménagement du Sud-Est de l'Angleterre (The South East of England development agency ou SEEDA) a repris la propriété et la gestion de Chatham Maritime à English partnerships en avril 1999.

14. South East England development agency (SEEDA), 2001, Chatham Maritime business plan 2001, Guilford, SEEDA. 
Les premières propositions d'aménagement des chantiers navals de Chatham ont été exposées en 1987, le dossier de réaménagement fournissant un cadre général pour les activités du site. Il a d'ailleurs été inclus dans le règlement d'urbanisme en 1992 (Medway towns local plan). La taille importante du site et l'appartenance du foncier au ministère de la Défense permettent de comprendre qu'il n'a pas été aussi difficile de lancer son aménagement que ceux d'autres projets dans lesquels la propriété du terrain était éclatée entre différents organismes. Son réaménagement a été entièrement financé par des subventions publiques. Ainsi, cette zone a bénéficié du statut de zone d'entreprise, entre 1986 et $1996^{15}$.

La section suivante examine le soutien apporté par Medway council (l'autorité locale) au projet de Chatham Maritime à la fois à travers les procédures administratives de son service d'urbanisme et de la stratégie de développement interne à la SEEDA. Ces deux démarches sont, dans une large mesure, déterminées par les règles établies par le gouvernement central. Par contraste avec les activités qui ont lieu au sein de la zone de régénération des mines de charbon, la consultation de la communauté locale a été très limitée.

Le Medway council plan ${ }^{16}$ stipule que Chatham Maritime devrait être une zone mixte (mixed-use zone), ce qui implique un centre d'innovation, un pôle d'activités d'entreprises, des équipements de loisirs, l'aménagement d'immeubles de bureaux et de logements résidentiels. Le rapport présenté au Service d'urbanisme de Medway council en 1998 expose quelques unes des objections faites à leurs propositions. Il y avait 17 lettres de riverains qui soutenaient le projet et une seulement qui s'y opposait. Cette objection était fondée sur l'hypothèse que le réaménagement nuirait aux centres urbains alentours ${ }^{17}$. Gravesend council exprima également son inquiétude quant à l'impact de ces projets sur le petit commerce local. Malgré ces objections, le Service de l'urbanisme et des transports approuva ces propositions lors d'une réunion le 4 aout 1998, en imposant toutefois 47 conditions d'aménagement, ayant trait aussi bien

15. Le statut de zone d'entreprise permet aux subventions qu'attribue le gouvernement aux investisseurs, développeurs et occupants de soutenir le réaménagement. L'aménagement des entreprises et des bureaux sur le site a bénéficié de cette source de financement.

16. Medway council, 1999, Medway council plan - 1999.

17. Medway council, 1998, Planning and transport committee $4^{\text {th }}$ August, Outline application for redevelopment of land buildings on Land South of Basin 1 Chatham Maritime. 
aux besoins en matière de transport qu'à la réduction de la taille du cinéma dont on avait proposé l'aménagement.

La régénération économique (economic regeneration) est un objectif clé pour les autorités locales et Medway council a cherché à attirer les investissements internes (inward investment). C'est le site de grandes compagnies telles que Marconi electronic eystems, Lucas diesel systems et Lloyd's colonial insurance. Ces dernières années la grande compagnie de télécommunication française Sagem s'est installée là; les compagnies japonaises Hochiki, Fuji seal et Fuji copian y ont implanté leur base européenne. Cependant, le projet d'entreprise de la SEEDA reconnait que:

L'aménagement n'a été que partiellement réussi. De nombreux employés de Chatham Maritime viennent de zones alentour et les employeurs, venus d'autres endroits de la zone de Medway towns, se sont relocalisés à Chatham Maritime. En soi, il n'est pas réaliste de penser que Chatham Maritime à lui seul puisse faire la différence. Ce qu'il peut et devrait faire cependant c'est jouer son rôle dans la perspective d'une stratégie économique plus vaste (wider economic strategy) (South East England development agency, 2001, Re: Chatham Maritime).

La reconnaissance explicite du fait que les aménagements phares devraient participer d'une stratégie économique d'ensemble et ne pas être une fin en soi est importante et contraste de manière frappante avec la stratégie de marketing (marketing) des brochures publicitaires distribuées par la SEEDA.

Le développement d'un commerce de détail et de loisirs novateurs fera venir des millions de visiteurs chaque année. Il s'agit d'un aménagement en parfait équilibre avec des zones entières du site qui sont dédiées à des projets de résidences, d'entreprises et de bureaux pour lesquels on a mis l'accent sur la recherche, l'éducation, la régénération, les technologies de l'information et l'innovation. De nombreuses entreprises ont déjà reconnu les opportunités offertes par Chatham Maritime. Mais il y a encore un énorme potentiel pour d'autres projets plus novateurs. La SEEDA dirige ce travail. Nous sommes là pour aider à ce que cela se fasse. (SEEDA, 2001, Re: Chatham Maritime, p. 1).

Les documents promotionnels traduisent le fait que l'aménagement de Chatham a été lancé avant l'établissement d'une base résidentielle. On peut estimer que c'est ce qui en a facilité l'aménagement, étant donné que l'obligation de consultation n'a pas été requise. Mais, comme Chatham Maritime est, en fait, un nouveau projet d'infrastructure, un véritable 
«esprit du lieu» a été difficile à créer jusqu'à présent. Considérons l'affirmation figurant dans le projet d'entreprise selon laquelle:

Chatham Maritime manque d'identité. Sur le plan physique, les voies d'accès au site ne sont pas indiquées... sur le plan social, il n'y pas vraiment de sentiment communautaire (sense of community) sur St Mary's Island et il y a très peu d'interaction entre les entreprises et les résidents (SEEDA, 2001, Re: Chatham Maritime).

Un représentant a exposé la position de l'autorité locale (Medway council) sur l'aménagement. Ses commentaires suggèrent que l'imposition d'une structure directive (top down) a été efficace pour mettre en place le projet et garantir qu'il se déroule dans les délais.

Le réaménagement de Chatham s'est fait selon une approche très directive (top down). Rien n'a été fait pour tenter d'entrer en contact avec les résidents puisque le site est fermé au public. Nous considérons que l'investissement fait par la SEEDA a réussi et nous travaillons avec les représentants de la SEEDA afin de nous assurer que nos préoccupations sont prises en compte. Il s'agit de trouver un équilibre ( $a$ balancing $a c t$ ). Nous devons essayer de garantir que le succès de l'aménagement de Chatham Maritime n'aura pas un impact négatif sur les centres urbains de Chatham et de Gillingham. Nous avons le sentiment que nous avons atteint le bon équilibre et que l'impact de ce qui est en train de se passer ne sera pas négatif. (Entretien, 22/03/01).

La stratégie de réaménagement de Chatham est conforme aux directives du gouvernement. Les objectifs locaux sont en quelque sorte harmonisés par le gouvernement. Au niveau global, l'essentiel de la stratégie vise ainsi à garantir un certain niveau de rendement et des résultats spécifiques qui prennent en compte différents éléments: le produit intérieur brut, le chômage, le pourcentage d'habitations construites sur un terrain préalablement aménagé, la productivité de la main d'œuvre, le pourcentage des résultats obtenus par la formation des adultes, la formation de nouvelles entreprises.

La redéveloppement a déjà eu un impact considérable. Il y a maintenant 200 unités résidentielles sur Saint Mary's Island et elles accueilleront 8000 personnes au final. Il y a certainement eu un effort pour promouvoir un bon design urbain, susciter l'émergence d'un esprit du lieu et mettre en place une politique intégrée des transports. Cependant aucun logement social n'a été construit sur le site. C'est l'une des conséquences du pouvoir exercé par le secteur privé dans les choix d'aménagement du 
site et de l'incapacité des groupes d'intérêt locaux à exiger des logements locatifs à bas prix.

On peut opposer cette absence de logements du secteur public à Chatham aux projets SRB de Douvres mieux adaptés pour répondre aux intérêts de la communauté locale. Le rôle dominant des promoteurs du secteur privé est un symptôme de l'absence de toute véritableforme de programme communautaire ou de mécanisme consultatif. Contrairement à l'East Kent coalfield partnership, les acteurs publics locaux n'ont aucune obligation de dialoguer avec les associations locales. Cela explique en partie pourquoi les responsables politiques qui travaillent sur ce projet peuvent adopter une perspective anticipatrice plutôt que réactive (ce qui est la norme parmi les responsables travaillant avec des associations locales). D'ailleurs, les accès au site sont fermés et les associations locales des quartiers de Brompton et de Gillingham ne sont pas représentées dans ces processus, malgré la proximité de ces villes. Dans ces conditions, il n'est pas surprenant qu'il n'y ait aucune représentation de la communauté locale.

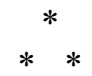

Nous avons examiné les manières dont le gouvernement du RoyaumeUni utilise les projets de réaménagement pour gérer les changements économiques et sociaux. Dans toutes les régions d'Angleterre, le gouvernement a mis en place des Agences de développement régional (Regional development agency ou RDA) afin de diriger différentes formes de réaménagement et d'investissement économique. La mise en place des RDA était censée constituer un moyen pour décentraliser le pouvoir en le déléguant aux régions. Cependant, les pratiques observées dans nos deux exemples suggèrent qu'il est très difficile de suivre localement des approches innovantes ou bien de dévier des directives gouvernementales, du fait des règles strictes imposées par le gouvernement et de la capacité limitée des acteurs des communautés locales à influencer les prises de décision. La capacité de l'État à façonner les résultats des politiques menées n'a pas été remise en cause.

Ces deux études de cas illustrent le fait que le type de réaménagement souhaité par les organismes d'État, tel qu'il est donné à lire dans les documents d'élaboration des politiques urbaines et les publicités, s'appuie largement sur le secteur privé. C'est une manifestation de la volonté du pou- 
voir central de se désinvestir partiellement de son image politique traditionnelle en cultivant une approche plus commerciale des questions locales. Les partenariats considérés comme réussis sont ceux qui donnent aux agents locaux de l'État un rôle de médiation active conciliant les intérêts de la communauté locale et ceux des entreprises. Le consensus est alors systématiquement recherché et les conflits gommés. L'analyse des documents de politique urbaine montre en fait à quel point les intérêts du monde des affaires ont primé sur toute tentative réelle d'implication de la communauté locale. À Chatham toutefois, dans les zones des anciennes mines de charbon, une tradition de militantisme politique a sans doute aidé les associations locales à s'assurer un rôle important dans le partenariat. 